\title{
Organ to Body Weight Ratio
}

National Cancer Institute

\section{Source}

National Cancer Institute. Organ to Body Weight Ratio. NCI Thesaurus. Code C90426.

A numeric comparison of the weight of an organ of interest to the weight of the body. 